\title{
Orbits and masses of twenty double stars discovered by Paul Couteau ${ }^{\star}$
}

\author{
J. A. Docobo ${ }^{1,2}$ and J. F. Ling ${ }^{1,2}$ \\ 1 Observatorio Astronómico Ramón María Aller, PO Box 197, Universidade de Santiago de Compostela, Spain \\ 2 Dpto. Matemática Aplicada, Universidade de Santiago de Compostela, Spain
}

Received 15 May 2003 / Accepted 25 June 2003

\begin{abstract}
In this article we present the orbits and masses of 20 double stars discovered by P. Couteau at the Nice Observatory (France), namely WDS 01450+2703 (COU 750), 03321+4340 (1688), 03423+3141 (691), 06503+2409 (768), $12409+2708(596), 14138+3059(606), 15390+2545$ (612), 16584+3943 (1289), 17075+3810 (1291), 17221+2310 (415), $18035+4032$ (1785), 18043+4206 (1786), 19089+3404 (1462), 19180+2012 (321), 20151+3742 (2416), $20216+1930$ (327 $\mathrm{AB}), 21593+4606$ (2138), 22077+2622 (537), 22307+1758 (234) and 23199+2844 (439). For two of these stars, COU 750 and COU 1289, these are the first orbits to be published other than IAU Circulars; for the others, previously published orbits are improved on by those presented here. Star masses were in fifteen cases calculated from Hipparcos parallax data, and in the other five from dynamical parallaxes; in all cases, the error is estimated. Some of the other physical and orbital properties of these stars are also discussed.
\end{abstract}

Key words. stars: binaries: general

\section{Introduction}

Starting in 1952 Paul Couteau discovered 2702 visual double stars using the 38,50 and $74 \mathrm{~cm}$ refractors of the Nice Observatory, France (Couteau 1999a, 1999b). Their components are on average of visual magnitude 9 and are separated by between $0 . ' 1$ and 0.5 . Several dozen have periods short enough for it already to have been possible to calculate orbits, and orbits for 72 of them are included in the Sixth Catalog of Orbits of Visual Binary Stars (Hartkopf \& Mason 2003) and the Catalog of Orbits and Ephemerides of Visual Double Stars (Docobo et al. 2001). The quality of these previously catalogued orbits according to the Sixth Catalog classification is of grade 2 in four cases, 3 in thirteen, 4 in twenty-eight, and 5 in twentyseven.

As part of the work on the astrometry, orbits and masses of double and multiple stars carried out at the Ramón María Aller Observatory at Santiago de Compostela, we have revised the previously catalogued orbits of eighteen Couteau binaries [WDS 03321+4340 (COU 1688), WDS 03423+3141 (COU 691), WDS 06503+2409 (COU 768), WDS 12409+2708 (596), WDS 14138+3059 (COU 606), WDS 15390+2545 (COU 612), WDS 17075+3810 (COU 1291), WDS 17221+2310 (COU 415), WDS 18035+4032

Send offprint requests to: J. A. Docobo or J. F. Ling, e-mail: oadoco, oafana@usc.es

* All the figures and tables are only available in electronic form at http://www. edpsciences.org
(COU 1785), WDS 18043+4206 (COU 1786), WDS 19089+3404 (COU 1462), WDS 19180+2012 (COU 321), WDS 20151+3742 (COU 2416), WDS 20216+1930 (COU 327 AB), WDS 21593+4606 (COU 2138), WDS 22077+2622 (COU 537), WDS 22307+1758 (COU 234) and WDS 23199+2844 (COU 439)] and have also calculated orbits for two others [WDS $01450+2703$ (COU 750) and WDS $16584+3943$ (COU 1289)]. In this paper we present these orbits together with total masses calculated from parallax data published in the Hipparcos and Tycho catalogues (ESA 1997) or, for five stars not featured in these catalogues, from dynamical parallaxes. In all cases, the error in the calculated mass has been estimated by analysis of the propagation of estimated observational error through the calculations.

The new orbits were calculated using Docobo's (1985) analytical method, and dynamical parallaxes using the BaizeRomaní algorithm (Heintz 1978). Unless otherwise stated, apparent magnitudes and spectral types are from the Washington Double Star Catalog (Mason et al. 2003; hereinafter "WDS"). All the orbits presented here have previously been announced in the Information Circulars of IAU Commission 26 (hereinafter IAUDS)

\section{Method}

One of the chief advantages of Docobo's analytical method (1985) is that it does not require knowledge of the areal constant. The method is based on a mapping from the interval $(0,2 \pi)$ into the family $\kappa$ of Keplerian orbits whose apparent 
orbits pass through three base points $\left(\theta_{i}, \rho_{i} ; t_{i}\right)(i=1,2,3)$. These base points are points belonging to regions of the orbit with a maximum of observational evidence in their favour, but do not necessarily coincide with actual observations. If the three base points do not belong to the same revolution, then the interval $(0,2 \pi)$ must be replaced by $(0,2 \pi n)$ where $n-1$ is the number of full revolutions between $t_{1}$ and $t_{3}$. The member of $\kappa$ that best fits the observational data measurements is chosen using a data weighting scheme similar to that used by Mason et al. (1999): weight 20 is given to speckle measurements with 4-6 m class telescopes, 10 to speckle data obtained with $2 \mathrm{~m}$ class telescopes, and 5 to poor-quality speckle and other interferometric measurements, and a maximum of 2.5 to visual observations, which are weighted using the formula

weight $=\frac{(n \times o b s)+t}{20}-0.5$

where $n$ is the number of nights on which observations were made, $o b s$ is a subjective note of observer experience $(1 \leq$ $o b s \leq 5$ ) and $t$ quantifies telescope class (45 for $2 \mathrm{~m}, 30$ for $1 \mathrm{~m}, 25$ for $0.5 \mathrm{~m}$, etc.).

\section{Results and discussion}

Table 1 lists, for each binary, the date of its discovery, the orbital elements calculated in this work (together with their estimated errors), and the precession correction used to refer position angles to the 2000.0 equinox. Each star is identified by its WDS number and its COU number (Couteau 1999a). Table 2 lists ephemerides for each binary for the period 2003-2012.

Table 3 lists WDS magnitudes in Cols. 3 and 4 and WDS spectral types in Col. 5; in Col. 6, the dynamical parallaxes calculated therefrom using the orbital periods and semiaxes major obtained in this work; in Cols. 7 and 8 the apparent magnitudes obtained by Hipparcos (ESA 1997), and in Col. 9 the corresponding trigonometric parallaxes (with their standard errors); and in Col. 10 the total mass of the binary ( \pm estimated standard error), as calculated from the Hipparcos parallax data or, in the cases of stars for which there are no Hipparcos data (indicated by asterisks in parentheses), from the corresponding dynamical parallax. The triple-star nature of WDS $20216+1930$ (COU 327 AB) (the third component is spectroscopic; Tokovinin 1994) is indicated in the table by a " $\mathrm{T}$ " in parentheses; the mass listed is the total mass of the three components.

Table 4 lists root mean squared residuals RMS and mean absolute residuals MA (observations with position angle residuals greater than $15^{\circ}$ with respect to all published orbits were ignored). For most of the stars considered, these results show that the new orbit fits the observational data better than previously published orbits. In the cases of COU 1688, COU 321 and COU 2138, the angular separation residuals are improved at the expense of the position angle residuals. In the case of COU $327 \mathrm{AB}$, neither the position angle residuals nor the angular separation residuals are better than those of a previous orbit of ours, but the present orbit implies a more plausible total mass than the other.
In Figs. 1-10 the new apparent orbits are drawn together with the observational data; micrometric observations are shown as dots and interferometric or CCD observations as stars. The epochs of the first and last of the observations used in the calculations are also shown. Arrows show the direction of motion, and the dashed line the line of nodes.

In what follows we comment on the results for each binary under a heading that includes its WDS and COU numbers, the quality of the orbit according to the classification of the Sixth Catalog of Orbits of Visual Binary Stars (Hartkopf \& Mason 2003) and the IAUDS in which the orbit was first announced.

\section{WDS 01450+2703, COU 750; grade 4} (IAUDS No. 138, 1999)

This is the first orbit for this star, for which there are no Hipparcos parallax data and the components of which are currently at minimum separation. Observations in the coming years will be important for confirmation of the orbit.

\section{WDS 03321+4340, COU 1688; grade 4} (IAUDS No. 141, 2000)

The first orbit for this short-period star was published by Baize (1993b). The semiaxis major of the new orbit, 0.'111, leads to a smaller RMS separation residual than is achieved by a rather similar orbit published by Manté (2000). However, since this star was first observed in 1978 and its components have not separated since 1986, all these orbits must be regarded as provisional until more observational data are available. In particular, both the new orbit and Manté's give a separation of 0 '. 11 for 2000.768 , on which date the WIYN 3.5 m telescope at Kitt Peak Observatory failed to resolve its components (Horch et al. 2002).

\section{WDS 03423+3141, COU 691; $\quad$ grade 4} (IAUDS No. 140, 2000)

Hartkopf et al.'s observation of 1996.8658, following passage through a separation minimum, showed the orbital period to be longer than the 31.42 years initially calculated by Baize (1993b). There are no Hipparcos parallax data for this star.

\section{WDS 06503+2409, COU 768; grade 3}

\section{(IAUDS No. 150, 2003)}

Revision of the orbit calculated by Baize (1988) for this star was called for by the separation residuals with respect to Baize's orbit having all been negative since 1984, and by its position angle residuals for the speckle measurements of 1995.1464 and 1996.8719 being quite unacceptably large. According to the new orbit, the star has already described a complete revolution since its discovery, and is now heading for a separation minimum. This orbit improves on the one previously announced by the authors in IAUDS No. 140 (Docobo \& Ling 2002a).

\section{WDS 12409+2708, COU 596; grade 3 (IAUDS No. 146, 2002)}

This star has nearly completed a whole revolution since its discovery. The period is thus considerably shorter than the 50 years estimated by Couteau (1999a). The orbit we 
have calculated is noteworthy for its eccentricity of 0.956 . The calculated dynamical parallax agrees very well with the Hipparcos measurement.

\section{WDS 14138+3059, COU 606; grade 3}

\section{(IAUDS No. 150, 2003)}

The present orbit improves on the one calculated for this pair of red dwarfs by Ling (1992). Although a whole revolution has been completed, speckle measurements for regions of the orbit for which none have so far been obtained would be desirable to enable calculation of a definitive solution.

\section{WDS 15390+2545, COU 612; grade 3 (IAUDS No. 136, 1998)}

This star has frequently been observed, and in recent years orbit calculations have been able to use numerous speckle measurements. The periods of the first and second orbits calculated (Baize 1993c; Aristidi et al. 1999) were 33 and 80 years, respectively. The new orbit (period 56 years) has slightly smaller RMS residuals than Aristidi et al.'s.

\section{WDS 16584+3943, COU 1289; grade 3 (IAUDS No. 139, 1999)}

The similar magnitudes of the components of this binary give rise to an uncertainty of $180^{\circ}$ in measured position angles, as a result of which we obtained and announced two contending solutions: a 16-year orbit of high eccentricity and a 37.6-year orbit of low eccentricity. The latter, however, must be rejected in view of Griffin's spectroscopic orbit (2003) who has recently shown that only the high-eccentricity orbit presented here is compatible with radial velocity measurements he performed in the period 1999-2002. Griffin's orbital elements lies within margins of our solution standard errors.

\section{WDS 17075+3810, COU 1291; grade 4 (IAUDS No. 147, 2002)}

The new orbit improves on those of Baize (1993c) and Docobo \& Ling (1998b). Some of the earlier reports of position angles appear to be quite seriously erroneous. According to the new orbit, the separation is currently decreasing towards a predicted minimum of $0{ }^{\prime} 05$. The calculated total mass is large for a G5 star.

\section{WDS 17221+2310, COU 415; grade 4 (IAUDS No. 149, 2003)}

The new orbit takes into account the increase in separation observed by Hartkopf et al. (1997) at 1995.4369. This leads to a longer period than the 60.10 years calculated by Baize (1993c), and to a total mass more in keeping with the spectral type.

\section{WDS 18035+4032, COU 1785; grade 4 (IAUDS No. 139, 1999)}

This orbit was first announced at the same time as Couteau's (1999a), for which the position angle residual for the speckle observation of 1995.6115 is excessive. A subsequent orbit of ours (Docobo \& Ling 2002a) has a more expectable distribution of residual signs, but poorer RMS residuals. There are no
Hipparcos parallax data for this star, which is currently leaving a separation minimum.

\section{WDS 18043+4206, COU 1786; grade 4 (IAUDS No. 141, 2000)}

This star was re-examined because the 40.6-year orbit calculated by Couteau (1993a) has shown systematic separation residuals for observations made since 1990, and also has residuals that are apparently excessive for some speckle position angles. The 18.75-year orbit suggested by Baize (1993b) proved to be erroneous.

\section{WDS 19089+3404, COU 1462; grade 3 (IAUDS No. 149, 2003)}

Horch et al.'s (2002) observation of 2000.767 suggests that the period must be rather longer than the 22.81 years predicted by Manté (1999b). The star is currently approaching a separation minimum, so measurements over the next few years should allow calculation of a definitive orbit.

\section{WDS 19180+2012, COU 321; grade 4 (IAUDS No. 146, 2002)}

The first orbit for this star was calculated by Baize (1993c). It is currently at a separation minimum, and seems likely to have a highly eccentric orbit of long period. Our previous orbit (Docobo \& Ling 1998a) does not account satisfactorily for the latest measurements, while an orbit proposed by Aristidi et al. (1999) implies a total mass that is too small for an A5 star.

\section{WDS 20151+3742, COU 2416; grade 5 (IAUDS No. 148, 2002)}

The speckle measurement of 1996.6986 (Hartkopf et al. 2000) ruled out Manté's (1999a) 61.68-year orbit. Both the new 57.61-year orbit and Couteau's (1999a) 31.50-year orbit fit the data well, especially as regards separation.

\section{WDS 20216+1930, COU $327 \mathrm{AB} ; \quad$ grade 4 (IAUDS No. 149, 2003)}

This is a multiple star system in which component $\mathrm{A}$ is a spectroscopic binary (Tokovinin 1994) and component C, of magnitude $\simeq 12$, lies about 4". from the others. Given the spectral type, and that the spectroscopic binary is of single-line type, the three components of the Aab-B system should have a total mass of 2.5-4.0 solar masses, which if the Hipparcos parallax measurement is accurate implies that the periods predicted previously, 35.00 years (Tokovinin 1994) and 34.48 years (Docobo \& Ling 2001b), must be too short. In calculating the new orbit we have ignored several highly unreliable observations obtained when the separation was less than 0 ' 10 . The period and semiaxis major of this orbit imply a parallax close to 5.9 mas.

\section{WDS 21593+4606, COU 2138; grade 5 (IAUDS No. 148, 2002)}

This orbit and Manté's (2002) orbit differ in their separation predictions. There are no spectral data for this star in WDS, but it is listed in SIMBAD (Wenger, M. et al. 2003) as of class K0. 


\section{WDS 22077+2622, COU 537; grade 3 (IAUDS No. 137, 1999)}

The speckle observations of 1994-96 (Hartkopf et al. 1997, 2000) show that the period of this star must be shorter than the 68.49 years predicted by Baize (1993b). These observations are also incompatible with a report that at 1998.663 the binary was not resolvable by the $2 \mathrm{~m}$ telescope at the Pic du Midi Observatory (Scardia et al. 2000). There are no Hipparcos parallax data for this star.

\section{WDS 22307+1758, COU 234; grade 4 (IAUDS No. 143, 2001)}

Baize's (1993a) prediction of a separation minimum of 0.'05 in 1995 was not fulfilled. According to the new orbit, this binary has a long period (134 years) and all speckle measurements have been performed near periastron; this orbit must therefore be considered as provisional, in spite of the marked improvement with respect to Baize's.

\section{WDS 23199+2844, COU 439; grade 4 (IAUDS No. 138, 1999)}

The new orbit for this star improves on Baize's (1993b) orbit, but must nevertheless be regarded as provisional because only the region around apoastron has been observed. Given the large mass it implies, its confirmation by future observations would suggest the presence of subcomponents in the system.

Acknowledgements. This article is a small tribute to Dr. Paul Couteau, who for many years afforded us invaluable collaboration and hospitality at the Nice and Pic du Midi Observatories. It has been supported by the Spanish Ministry of Science and Technology under Project AYA2001-3073, and by the Xunta de Galicia under Project PGIDIT02PXIC 24301PN.

\section{References}

Aristidi, E., Prieur, J.-L., Scardia, M., et al. 1999, A\&AS, 134, 545 Baize, P. 1988, IAUDS, Inf. Circ. 106 Baize, P. 1993a, IAUDS, Inf. Circ. 120 Baize, P. 1993b, IAUDS, Inf. Circ. 121 Baize, P. 1993c, A\&AS, 99, 205 Couteau P. 1993a, IAUDS, Inf. Circ. 119
Couteau P. 1999a, Catalogue de 2700 Étoiles Doubles COU, Observatoire de la Côte D'Azur, Département Augustin Fresnel UMR 6528, 3ème edn.

Couteau P. 1999b, IAUDS, Inf. Circ. 138

Docobo, J. A. 1985, Cel. Mech., 36, 143

Docobo, J. A., \& Ling, J. F. 1998a, IAUDS, Inf. Circ. 134

Docobo, J. A., \& Ling, J. F. 1998b, IAUDS, Inf. Circ. 136

Docobo, J. A., \& Ling, J. F. 1999a, IAUDS, Inf. Circ. 137

Docobo, J. A., \& Ling, J. F. 1999b, IAUDS, Inf. Circ. 138

Docobo, J. A., \& Ling, J. F. 1999c, IAUDS, Inf. Circ. 139

Docobo, J. A., \& Ling, J. F. 2000a, IAUDS, Inf. Circ. 140

Docobo, J. A., \& Ling, J. F. 2000b, IAUDS, Inf. Circ. 141

Docobo, J. A., \& Ling, J. F. 2001a, IAUDS, Inf. Circ. 143

Docobo, J. A., \& Ling, J. F. 2001b, IAUDS, Inf. Circ. 145

Docobo, J. A., \& Ling, J. F. 2002a, IAUDS, Inf. Circ. 146

Docobo, J. A., \& Ling, J. F. 2002b, IAUDS, Inf. Circ. 147

Docobo, J. A., \& Ling, J. F. 2002c, IAUDS, Inf. Circ. 148

Docobo, J. A., \& Ling, J. F. 2003a, IAUDS, Inf. Circ. 149

Docobo, J. A., \& Ling, J. F. 2003b, IAUDS, Inf. Circ. 150

Docobo, J. A., Ling, J. F., Prieto, C., et al. 2001, AcA, 51, 353

Griffin, R. G. 2003, Obs, in press

ESA 1997, The Hipparcos and Tycho Catalogues, ESA Pub. Div. ESTEC, Noordwijk, The Netherlands

Hartkopf, W. I., \& Mason, B. D. 2003, http://ad.usno.navy.mil/wds/orb6.html

Hartkopf, W. I., McAlister, H. A., Mason, B. D., et al. 1997, AJ, 114, 1639

Hartkopf, W. I., Mason, B. D., McAlister, H. A., et al. 2000, AJ, 119, 3084

Heintz, W. D. 1978, Double Stars (Dordrecht: Reidel)

Horch, E. P., Robinson, S. E., Meyer, R. D., et al. 2002, AJ, 123, 3442

Ling, J. F. 1992, AN, 313, 91

Manté, R. 1999a, IAUDS, Inf. Circ. 137

Manté, R. 1999b, IAUDS, Inf. Circ. 138

Manté, R. 2000, IAUDS, Inf. Circ. 141

Manté, R. 2002, IAUDS, Inf. Circ. 148

Mason, B. D., Douglass, G. G., \& Hartkopf, W. I. 1999, AJ, 117, 1023

Mason B. D.; Wycoff, G., \& Hartkopf, W. I. 2003, http://ad.usno.navy.mil/wds/wds.html

Scardia, M., Prieur, J.-L., Aristidi, E., \& Koechlin, L. 2000, ApJS, 131,561

Wenger, M., Ochsenbein, F., Egret, D., et al. 2003, SIMBAD astronomical data base, http://simbad.u-strasbg.fr/

Tokovinin, A. A. 1994, AstL, 20,435 
J. A. Docobo and J. F. Ling: Orbits and masses of 20 double stars discovered by Couteau, Online Material $p 1$

\section{Online Material}


Table 1. Orbital elements.

\begin{tabular}{lccccc}
\hline \hline & $01450+2703$ & $03321+4340$ & $03423+3141$ & $06503+2409$ & $12409+2708$ \\
& COU 750 & COU 1688 & COU 691 & COU 768 & COU 596 \\
\hline Disc. date & 1972.80 & 1978.85 & 1972.05 & 1972.18 & 1971.30 \\
$P(\mathrm{yr})$ & $40.0 \pm 2.5$ & $9.26 \pm 0.2$ & $36.52 \pm 2.0$ & $25.95 \pm 0.5$ & $33.30 \pm 1.0$ \\
$T(\mathrm{yr})$ & $2002.46 \pm 1.5$ & $2004.64 \pm 0.2$ & $2003.74 \pm 1.0$ & $2008.38 \pm 0.5$ & $2022.29 \pm 0.2$ \\
$e$ & $0.778 \pm 0.100$ & $0.139 \pm 0.010$ & $0.600 \pm 0.070$ & $0.467 \pm 0.020$ & $0.956 \pm 0.010$ \\
$a(\operatorname{arcsec})$ & $0.298 \pm 0.010$ & $0.111 \pm 0.008$ & $0.167 \pm 0.010$ & $0.118 \pm 0.005$ & $0.108 \pm 0.005$ \\
$i(\mathrm{deg})$ & $81.9 \pm 2.5$ & $22.9 \pm 3.0$ & $73.9 \pm 1.5$ & $144.2 \pm 3.5$ & $132.1 \pm 1.0$ \\
$\Omega(\mathrm{deg})$ & $27.4 \pm 1.0$ & $9.0 \pm 4.0$ & $52.7 \pm 1.0$ & $48.8 \pm 1.0$ & $54.0 \pm 8.0$ \\
$\omega(\mathrm{deg})$ & $263.2 \pm 3.0$ & $281.2 \pm 5.0$ & $252.0 \pm 2.0$ & $45.6 \pm 0.5$ & $40.3 \pm 1.0$ \\
Precession $(\mathrm{deg})$ & 0.0028 & 0.0062 & 0.0054 & 0.0060 & -0.0011 \\
\hline \hline
\end{tabular}

\begin{tabular}{lccccc}
\hline \hline & $\begin{array}{c}14138+3059 \\
\text { COU 606 }\end{array}$ & $\begin{array}{c}15390+2545 \\
\text { COU 612 }\end{array}$ & $\begin{array}{c}16584+3943 \\
\text { COU 1289 }\end{array}$ & $\begin{array}{c}17075+3810 \\
\text { COU 1291 }\end{array}$ & $\begin{array}{c}17221+2310 \\
\text { COU 415 }\end{array}$ \\
\hline Disc. date & 1971.37 & 1971.41 & 1975.44 & 1975.33 & 1969.47 \\
$P(\mathrm{yr})$ & $24.21 \pm 0.2$ & $56.00 \pm 1.0$ & $15.99 \pm 0.2$ & $51.03 \pm 3.0$ & $236.36 \pm 15.0$ \\
$T(\mathrm{yr})$ & $2009.22 \pm 0.05$ & $2027.07 \pm 0.2$ & $2001.05 \pm 0.5$ & $2011.66 \pm 2.0$ & $2224.65 \pm 1.0$ \\
$e$ & $0.295 \pm 0.01$ & $0.405 \pm 0.010$ & $0.832 \pm 0.100$ & $0.331 \pm 0.080$ & $0.696 \pm 0.050$ \\
$a(\operatorname{arcsec})$ & $0.158 \pm 0.002$ & $0.212 \pm 0.005$ & $0.076 \pm 0.008$ & $0.174 \pm 0.008$ & $0.532 \pm 0.020$ \\
$i(\mathrm{deg})$ & $160.5 \pm 5.0$ & $137.7 \pm 1.0$ & $116.4 \pm 5.0$ & $62.6 \pm 2.0$ & $153.8 \pm 2.0$ \\
$\Omega(\mathrm{deg})$ & $90.6 \pm 10.0$ & $164.4 \pm 1.0$ & $58.2 \pm 12.0$ & $121.2 \pm 1.0$ & $175.6 \pm 3.0$ \\
$\omega(\mathrm{deg})$ & $225.8 \pm 15.0$ & $131.2 \pm 2.0$ & $149.1 \pm 20.0$ & $278.9 \pm 1.0$ & $161.3 \pm 4.0$ \\
Precession (deg) & -0.0036 & -0.0051 & -0.0070 & -0.0069 & -0.0060 \\
\hline \hline
\end{tabular}

\begin{tabular}{lccccc}
\hline \hline & $\begin{array}{c}18035+4032 \\
\text { COU 1785 }\end{array}$ & $\begin{array}{c}18043+4206 \\
\text { COU 1786 }\end{array}$ & $\begin{array}{c}19089+3404 \\
\text { COU 1462 }\end{array}$ & $\begin{array}{c}19180+2012 \\
\text { COU 321 }\end{array}$ & $\begin{array}{c}20151+3742 \\
\text { COU 2416 }\end{array}$ \\
\hline & & & & & \\
Disc. date & 1978.42 & 1979.45 & 1976.53 & 1968.69 & 1986.86 \\
$P(\mathrm{yr})$ & $34.83 \pm 2.5$ & $46.95 \pm 2.0$ & $23.39 \pm 0.4$ & $354.12 \pm 20.0$ & $57.61 \pm 4.0$ \\
$T(\mathrm{yr})$ & $2032.50 \pm 0.5$ & $2038.34 \pm 1.0$ & $2017.25 \pm 0.2$ & $2349.40 \pm 2.0$ & $2053.92 \pm 0.5$ \\
$e$ & $0.413 \pm 0.200$ & $0.190 \pm 0.050$ & $0.682 \pm 0.020$ & $0.776 \pm 0.100$ & $0.541 \pm 0.050$ \\
$a(\operatorname{arcsec})$ & $0.130 \pm 0.008$ & $0.133 \pm 0.005$ & $0.313 \pm 0.008$ & $0.484 \pm 0.020$ & $0.234 \pm 0.010$ \\
$i(\mathrm{deg})$ & $82.4 \pm 1.0$ & $46.9 \pm 1.0$ & $83.6 \pm 1.0$ & $101.0 \pm 2.0$ & $33.9 \pm 3.0$ \\
$\Omega(\mathrm{deg})$ & $51.7 \pm 1.0$ & $36.0 \pm 2.5$ & $38.6 \pm 1.0$ & $142.2 \pm 3.0$ & $128.5 \pm 3.0$ \\
$\omega(\mathrm{deg})$ & $195.0 \pm 9.0$ & $166.5 \pm 7.0$ & $272.7 \pm 2.0$ & $188.4 \pm 5.0$ & $229.0 \pm 5.0$ \\
Precession $(\mathrm{deg})$ & -0.0074 & -0.0075 & -0.0065 & -0.0056 & -0.0047 \\
\hline \hline
\end{tabular}

\begin{tabular}{lccccc}
\hline \hline & $20216+1930$ & $21593+4606$ & $22077+2622$ & $22307+1758$ & $23199+2844$ \\
& COU 327 AB & COU 2138 & COU 537 & COU 234 & COU 439 \\
\hline & & & & & \\
Disc. date & 1968.86 & 1982.82 & 1970.80 & 1967.66 & 1969.73 \\
$P(\mathrm{yr})$ & $62.76 \pm 2.0$ & $39.69 \pm 2.0$ & $45.43 \pm 0.8$ & $134.0 \pm 10.0$ & $64.79 \pm 3.0$ \\
$T(\mathrm{yr})$ & $2048.14 \pm 0.2$ & $2003.71 \pm 1.5$ & $2039.10 \pm 0.1$ & $2126.00 \pm 1.0$ & $2021.76 \pm 8.0$ \\
$e$ & $0.576 \pm 0.020$ & $0.176 \pm 0.050$ & $0.578 \pm 0.010$ & $0.523 \pm 0.050$ & $0.312 \pm 0.080$ \\
$a(\operatorname{arcsec})$ & $0.137 \pm 0.010$ & $0.220 \pm 0.005$ & $0.180 \pm 0.010$ & $0.333 \pm 0.015$ & $0.195 \pm 0.008$ \\
$i(\mathrm{deg})$ & $80.3 \pm 1.0$ & $116.3 \pm 2.0$ & $39.5 \pm 1.8$ & $110.7 \pm 2.0$ & $53.6 \pm 3.0$ \\
$\Omega(\mathrm{deg})$ & $65.9 \pm 1.0$ & $10.6 \pm 1.0$ & $63.4 \pm 2.3$ & $127.1 \pm 3.0$ & $12.0 \pm 4.0$ \\
$\omega(\operatorname{deg})$ & $309.3 \pm 2.0$ & $204.6 \pm 15.0$ & $94.9 \pm 3.6$ & $197.0 \pm 5.0$ & $45.3 \pm 8.0$ \\
Precession $(\mathrm{deg})$ & -0.0048 & -0.0041 & -0.0029 & -0.0022 & -0.0011 \\
\hline \hline
\end{tabular}


Table 2. Ephemerides.

\begin{tabular}{ccccccccccc}
\hline \hline$t$ & \multicolumn{2}{c}{$01450+2703$} & \multicolumn{2}{c}{$03321+4340$} & \multicolumn{2}{c}{$03423+3141$} & \multicolumn{2}{c}{$06503+2409$} & \multicolumn{2}{c}{$12409+2708$} \\
& $\theta\left(^{\circ}\right)$ & $\rho\left({ }^{\prime \prime}\right)$ & $\theta\left({ }^{\circ}\right)$ & $\rho\left({ }^{\prime \prime}\right)$ & $\theta\left({ }^{\circ}\right)$ & $\rho\left({ }^{\prime \prime}\right)$ & $\theta\left(^{\circ}\right)$ & $\rho\left({ }^{\prime \prime}\right)$ & $\theta\left({ }^{\circ}\right)$ & $\rho\left(^{\prime \prime}\right)$ \\
\hline & & & & & & & & & & \\
2003.0 & 20.0 & 0.060 & 209.6 & 0.105 & 244.6 & 0.058 & 128.3 & 0.105 & 206.3 & 0.185 \\
2004.0 & 27.9 & 0.136 & 255.7 & 0.091 & 304.3 & 0.020 & 114.6 & 0.097 & 205.6 & 0.186 \\
2005.0 & 30.8 & 0.176 & 310.9 & 0.091 & 32.5 & 0.051 & 98.5 & 0.089 & 204.8 & 0.186 \\
2006.0 & 32.8 & 0.199 & 358.0 & 0.103 & 45.3 & 0.091 & 79.2 & 0.081 & 204.1 & 0.185 \\
2007.0 & 34.4 & 0.211 & 35.7 & 0.112 & 50.8 & 0.120 & 55.7 & 0.072 & 203.4 & 0.183 \\
2008.0 & 36.0 & 0.218 & 69.7 & 0.115 & 54.4 & 0.141 & 24.3 & 0.061 & 202.6 & 0.181 \\
2009.0 & 37.4 & 0.220 & 102.4 & 0.116 & 57.2 & 0.155 & 340.9 & 0.054 & 201.9 & 0.177 \\
2010.0 & 38.9 & 0.219 & 134.2 & 0.118 & 59.6 & 0.164 & 298.0 & 0.063 & 201.1 & 0.173 \\
2011.0 & 40.4 & 0.215 & 165.6 & 0.117 & 61.8 & 0.169 & 270.4 & 0.081 & 200.2 & 0.168 \\
2012.0 & 42.0 & 0.209 & 199.6 & 0.108 & 63.9 & 0.171 & 253.4 & 0.100 & 199.3 & 0.162 \\
\hline
\end{tabular}

\begin{tabular}{ccccccccccc}
\hline \hline$t$ & \multicolumn{2}{c}{$14138+3059$} & \multicolumn{2}{c}{$15390+2545$} & \multicolumn{2}{c}{$16584+3943$} & \multicolumn{2}{c}{$17075+3810$} & \multicolumn{2}{c}{$17221+2310$} \\
& $\theta\left({ }^{\circ}\right)$ & $\rho\left({ }^{\prime \prime}\right)$ & $\theta\left({ }^{\circ}\right)$ & $\rho\left({ }^{\prime \prime}\right)$ & $\theta\left({ }^{\circ}\right)$ & $\rho\left({ }^{\prime \prime}\right)$ & $\theta\left(^{\circ}\right)$ & $\rho\left({ }^{\prime \prime}\right)$ & $\theta\left({ }^{\circ}\right)$ & $\rho\left({ }^{\prime \prime}\right)$ \\
\hline & & & & & & & & & & \\
2003.0 & 348.2 & 0.163 & 193.5 & 0.268 & 100.2 & 0.046 & 301.0 & 0.164 & 273.2 & 0.288 \\
2004.0 & 334.8 & 0.154 & 190.8 & 0.270 & 90.1 & 0.068 & 304.6 & 0.155 & 270.0 & 0.299 \\
2005.0 & 319.8 & 0.144 & 188.2 & 0.271 & 84.5 & 0.085 & 308.8 & 0.144 & 267.0 & 0.311 \\
2006.0 & 302.6 & 0.134 & 185.5 & 0.271 & 80.8 & 0.099 & 313.7 & 0.131 & 264.2 & 0.323 \\
2007.0 & 282.7 & 0.125 & 182.8 & 0.270 & 77.8 & 0.110 & 319.9 & 0.114 & 261.6 & 0.334 \\
2008.0 & 259.4 & 0.116 & 180.1 & 0.268 & 75.4 & 0.118 & 328.2 & 0.096 & 259.2 & 0.346 \\
2009.0 & 232.7 & 0.109 & 177.4 & 0.265 & 73.2 & 0.123 & 340.6 & 0.078 & 257.0 & 0.357 \\
2010.0 & 204.0 & 0.108 & 174.5 & 0.262 & 71.1 & 0.126 & 0.2 & 0.062 & 254.9 & 0.369 \\
2011.0 & 176.4 & 0.114 & 171.6 & 0.257 & 69.1 & 0.125 & 29.2 & 0.054 & 252.9 & 0.380 \\
2012.0 & 152.8 & 0.125 & 168.6 & 0.251 & 67.0 & 0.121 & 59.6 & 0.059 & 251.0 & 0.391 \\
\hline
\end{tabular}

\begin{tabular}{|c|c|c|c|c|c|c|c|c|c|c|}
\hline \multirow[t]{2}{*}{$t$} & \multicolumn{2}{|c|}{$18035+4032$} & \multicolumn{2}{|c|}{$18043+4206$} & \multicolumn{2}{|c|}{$19089+3404$} & \multicolumn{2}{|c|}{$19180+2012$} & \multicolumn{2}{|c|}{$20151+3742$} \\
\hline & $\theta\left({ }^{\circ}\right)$ & $\rho\left({ }^{\prime \prime}\right)$ & $\theta\left(^{\circ}\right)$ & $\rho\left({ }^{\prime \prime}\right)$ & $\theta\left(^{\circ}\right)$ & $\rho\left({ }^{\prime \prime}\right)$ & $\theta\left(^{\circ}\right)$ & $\rho\left({ }^{\prime \prime}\right)$ & $\theta\left(^{\circ}\right)$ & $\rho\left({ }^{\prime \prime}\right)$ \\
\hline 2003.0 & 333.5 & 0.021 & 316.0 & 0.095 & 78.1 & 0.088 & 265.2 & 0.036 & 106.7 & 0.187 \\
\hline 2004.0 & 14.6 & 0.035 & 325.7 & 0.099 & 99.6 & 0.066 & 240.1 & 0.033 & 112.9 & 0.205 \\
\hline 2005.0 & 29.7 & 0.054 & 334.5 & 0.105 & 133.0 & 0.059 & 215.0 & 0.036 & 118.2 & 0.222 \\
\hline 2006.0 & 36.7 & 0.074 & 342.4 & 0.111 & 163.7 & 0.071 & 196.7 & 0.044 & 122.7 & 0.237 \\
\hline 2007.0 & 40.8 & 0.093 & 349.4 & 0.117 & 181.8 & 0.096 & 185.0 & 0.055 & 126.7 & 0.251 \\
\hline 2008.0 & 43.5 & 0.110 & 355.6 & 0.124 & 192.2 & 0.125 & 177.3 & 0.068 & 130.4 & 0.263 \\
\hline 2009.0 & 45.5 & 0.126 & 1.3 & 0.131 & 198.6 & 0.154 & 172.1 & 0.081 & 133.7 & 0.274 \\
\hline 2010.0 & 47.1 & 0.140 & 6.4 & 0.137 & 203.0 & 0.181 & 168.3 & 0.094 & 136.7 & 0.284 \\
\hline 2011.0 & 48.4 & 0.152 & 11.0 & 0.142 & 206.3 & 0.206 & 165.5 & 0.107 & 139.6 & 0.293 \\
\hline 2012.0 & 49.6 & 0.162 & 15.4 & 0.147 & 208.9 & 0.225 & 163.3 & 0.120 & 142.3 & 0.301 \\
\hline \multirow[t]{2}{*}{$t$} & \multicolumn{2}{|c|}{$20216+1930$} & \multicolumn{2}{|c|}{$21593+4606$} & \multicolumn{2}{|c|}{$22077+2622$} & \multicolumn{2}{|c|}{$22307+1758$} & \multicolumn{2}{|c|}{$23199+2844$} \\
\hline & $\theta\left({ }^{\circ}\right)$ & $\rho\left({ }^{\prime \prime}\right)$ & $\theta\left(^{\circ}\right)$ & $\rho\left({ }^{\prime \prime}\right)$ & $\theta\left(^{\circ}\right)$ & $\rho\left({ }^{\prime \prime}\right)$ & $\theta\left(^{\circ}\right)$ & $\rho\left({ }^{\prime \prime}\right)$ & $\theta\left(^{\circ}\right)$ & $\rho\left({ }^{\prime \prime}\right)$ \\
\hline 2003.0 & 203.6 & 0.046 & 183.7 & 0.177 & 288.2 & 0.180 & 187.7 & 0.091 & 283.5 & 0.134 \\
\hline 2004.0 & 209.8 & 0.053 & 177.1 & 0.164 & 293.0 & 0.186 & 178.3 & 0.105 & 290.3 & 0.132 \\
\hline 2005.0 & 214.5 & 0.060 & 169.2 & 0.147 & 297.6 & 0.191 & 171.0 & 0.119 & 297.2 & 0.131 \\
\hline 2006.0 & 218.1 & 0.068 & 158.9 & 0.127 & 301.9 & 0.196 & 165.4 & 0.135 & 304.1 & 0.131 \\
\hline 2007.0 & 221.0 & 0.075 & 144.8 & 0.107 & 306.0 & 0.200 & 161.0 & 0.150 & 311.0 & 0.132 \\
\hline 2008.0 & 223.4 & 0.083 & 125.1 & 0.092 & 310.0 & 0.204 & 157.4 & 0.166 & 317.7 & 0.134 \\
\hline 2009.0 & 225.3 & 0.090 & 100.9 & 0.088 & 313.8 & 0.207 & 154.4 & 0.182 & 324.2 & 0.137 \\
\hline 2010.0 & 227.0 & 0.097 & 77.9 & 0.097 & 317.6 & 0.210 & 152.0 & 0.198 & 330.5 & 0.140 \\
\hline 2011.0 & 228.5 & 0.104 & 60.4 & 0.114 & 321.2 & 0.212 & 149.8 & 0.213 & 336.4 & 0.143 \\
\hline 2012.0 & 229.8 & 0.111 & 48.1 & 0.137 & 324.7 & 0.214 & 148.0 & 0.228 & 342.2 & 0.145 \\
\hline
\end{tabular}


J. A. Docobo and J. F. Ling: Orbits and masses of 20 double stars discovered by Couteau, Online Material p 4

Table 3. Stellar data.

\begin{tabular}{|c|c|c|c|c|c|c|c|c|c|}
\hline \multicolumn{2}{|c|}{ Star } & \multicolumn{3}{|c|}{ WDS. } & \multirow{2}{*}{$\begin{array}{c}\text { Present work } \\
\pi \text { (dyn.) } \\
\text { (arcsec) }\end{array}$} & \\
\hline WDS & $\mathrm{COU}$ & $m_{\mathrm{A}}$ & $m_{\mathrm{B}}$ & $\begin{array}{l}\text { Spectral } \\
\text { type }\end{array}$ & & $m_{\mathrm{A}}$ & $m_{\mathrm{B}}$ & $\begin{array}{l}\pi \text { (trig.) } \\
\text { (arcsec) }\end{array}$ & $\begin{array}{c}\sum M_{\odot} \\
\text { (Solar mass) }\end{array}$ \\
\hline $01450+2703$ & 750 & 9.7 & 9.7 & $\mathrm{~K} 2$ & 0.02181 & - & - & - & $1.59 \pm 0.34\left(^{*}\right)$ \\
\hline $03321+4340$ & 1688 & 8.7 & 8.9 & G5 & 0.02048 & - & - & $0.01858 \pm 0.00109$ & $2.49 \pm 0.70$ \\
\hline $03423+3141$ & 691 & 9.0 & 9.0 & F8 & 0.01158 & - & - & - & $2.25 \pm 0.75(*)$ \\
\hline $06503+2409$ & 768 & 8.34 & 9.12 & F5 & 0.00974 & 8.883 & 9.668 & $0.00864 \pm 0.00126$ & $3.78 \pm 1.73$ \\
\hline $12409+2708$ & 596 & 8.5 & 8.7 & F5V & 0.00707 & - & - & $0.00705 \pm 0.00092$ & $3.24 \pm 1.36$ \\
\hline $14138+3059$ & 606 & 10.73 & 11.32 & M0 & 0.01577 & 10.734 & 11.316 & $0.01721 \pm 0.00163$ & $1.32 \pm 0.38$ \\
\hline $15390+2545$ & 612 & 8.16 & 8.28 & G5 & 0.00990 & 9.150 & 9.035 & $0.01104 \pm 0.00122$ & $2.26 \pm 0.77$ \\
\hline $16584+3943$ & 1289 & 8.4 & 8.4 & G0 & 0.00820 & - & - & $0.00843 \pm 0.00080$ & $2.87 \pm 1.22$ \\
\hline $17075+3810$ & 1291 & 8.57 & 8.88 & G5 & 0.00882 & 9.295 & 9.615 & $0.00880 \pm 0.00082$ & $2.97 \pm 0.99$ \\
\hline $17221+2310$ & 415 & 8.29 & 9.38 & F5 & 0.01023 & 8.747 & 9.833 & $0.00901 \pm 0.00122$ & $3.68 \pm 1.62$ \\
\hline $18035+4032$ & 1785 & 8.7 & 8.9 & F5 & 0.00871 & - & - & - & $2.74 \pm 0.99(*)$ \\
\hline $18043+4206$ & 1786 & 7.84 & 8.62 & F0 & 0.00664 & 8.334 & 9.112 & $0.00659 \pm 0.00064$ & $3.73 \pm 1.21$ \\
\hline $19089+3404$ & 1462 & 9.42 & 9.57 & K8 & 0.03339 & 10.216 & 10.364 & $0.03437 \pm 0.00130$ & $1.38 \pm 0.19$ \\
\hline $19180+2012$ & 321 & 8.0 & 8.0 & A5 & 0.00599 & 8.919 & 8.918 & $0.00597 \pm 0.00097$ & $4.25 \pm 2.19$ \\
\hline $20151+3742$ & 2416 & 8.0 & 8.5 & F0V & 0.01120 & - & - & - & $2.75 \pm 0.90(*)$ \\
\hline $20216+1930$ & $327 \mathrm{AB}$ & 9.1 & 9.1 & F8 & - & 8.489 & - & $0.00455 \pm 0.00196$ & $3.30 \pm 0.70(\mathrm{~T})$ \\
\hline $21593+4606$ & 2138 & 9.5 & 9.8 & - & 0.01535 & - & - & $0.01532 \pm 0.00114$ & $1.88 \pm 0.48$ \\
\hline $22077+2622$ & 537 & 8.6 & 8.8 & G0 & 0.01032 & - & - & - & $2.57 \pm 0.87(*)$ \\
\hline $22307+1758$ & 234 & 8.50 & 9.04 & F8 & 0.00913 & 9.676 & 9.132 & $0.00823 \pm 0.00110$ & $3.69 \pm 1.66$ \\
\hline $23199+2844$ & 439 & 8.68 & 9.38 & F8 & 0.00881 & 9.240 & 9.944 & $0.00717 \pm 0.00137$ & $4.79 \pm 2.84$ \\
\hline
\end{tabular}


Table 4. Statistical results.

\begin{tabular}{|c|c|c|c|c|c|c|}
\hline \multicolumn{2}{|c|}{ Star } & \multirow[t]{2}{*}{ Authors } & \multicolumn{2}{|c|}{ RMS } & \multicolumn{2}{|c|}{ MA } \\
\hline WDS & $\mathrm{COU}$ & & $\Delta \theta\left(^{\circ}\right)$ & $\Delta \rho\left({ }^{\prime \prime}\right)$ & $\Delta \theta\left(^{\circ}\right)$ & $\Delta \rho\left(^{\prime \prime}\right)$ \\
\hline $01450+2703$ & 750 & Docobo \& Ling (this paper) & 6.596 & 0.027 & 1.159 & 0.58 \\
\hline \multirow[t]{3}{*}{$03321+4340$} & 1688 & Docobo \& Ling (this paper) & 8.102 & 0.020 & 4.353 & 0.024 \\
\hline & & Manté (2000) & 7.896 & 0.023 & 4.259 & 0.029 \\
\hline & & Baize (1993b) & 8.674 & 0.013 & 5.291 & 0.015 \\
\hline \multirow[t]{2}{*}{$03423+3141$} & 691 & Docobo \& Ling (this paper) & 16.129 & 0.029 & 3.692 & 0.048 \\
\hline & & Baize (1993b) & 25.119 & 0.049 & 5.225 & 0.083 \\
\hline \multirow[t]{2}{*}{$06503+2409$} & 768 & Docobo \& Ling (this paper) & 6.206 & 0.023 & 1.675 & $\overline{0.042}$ \\
\hline & & Baize (1988) & 36.604 & 0.142 & 7.795 & 0.194 \\
\hline $12409+2708$ & 596 & Docobo \& Ling (this paper) & 9.869 & 0.022 & 1.687 & 0.058 \\
\hline \multirow[t]{2}{*}{$14138+3059$} & 606 & Docobo \& Ling (this paper) & 17.177 & 0.047 & 3.024 & 0.078 \\
\hline & & Ling (1992) & 17.352 & 0.053 & 3.096 & 0.087 \\
\hline \multirow[t]{3}{*}{$15390+2545$} & 612 & Docobo \& Ling (this paper) & 8.470 & 0.036 & 1.524 & 0.052 \\
\hline & & Aristidi et al. (1999) & 9.898 & 0.052 & 1.726 & 0.081 \\
\hline & & Baize (1993c) & 16.016 & 0.110 & 2.955 & 0.130 \\
\hline $16584+3943$ & 1289 & Docobo \& Ling ; (this paper) & 7.580 & 0.072 & 1.900 & 0.087 \\
\hline \multirow[t]{3}{*}{$17075+3810$} & 1291 & Docobo \& Ling (this paper) & 11.787 & 0.033 & 2.873 & $\overline{0.048}$ \\
\hline & & Docobo \& Ling (1998b) & 15.318 & 0.034 & 3.460 & 0.051 \\
\hline & & Baize (1993b) & 30.585 & 0.103 & 4.746 & 0.158 \\
\hline \multirow[t]{2}{*}{$17221+2310$} & 415 & Docobo \& Ling (this paper) & 12.827 & 0.069 & 2.611 & 0.073 \\
\hline & & Baize $(1993 \mathrm{c})$ & 16.682 & 0.070 & 3.689 & 0.079 \\
\hline \multirow[t]{3}{*}{$18035+4032$} & 1785 & Docobo \& Ling (this paper) & 9.988 & 0.027 & 2.803 & 0.046 \\
\hline & & Docobo \& Ling (2002a) & 12.039 & 0.048 & 3.143 & 0.069 \\
\hline & & Couteau (1999a) & 35.876 & 0.055 & 7.439 & 0.075 \\
\hline \multirow[t]{3}{*}{$18043+4206$} & 1786 & Docobo \& Ling (this paper) & 8.580 & 0.024 & 1.560 & 0.051 \\
\hline & & Baize (1993b) & 27.710 & 0.058 & 4.884 & 0.132 \\
\hline & & Couteau $(1993 a)$ & 9.974 & 0.047 & 1.916 & 0.099 \\
\hline \multirow[t]{2}{*}{$19089+3404$} & 1462 & Docobo \& Ling (this paper) & 7.763 & 0.029 & 1.228 & 0.059 \\
\hline & & Manté (1999b) & 8.556 & 0.051 & 1.541 & 0.101 \\
\hline \multirow[t]{4}{*}{$19180+2012$} & 321 & Docobo \& Ling (this paper) & 12.605 & 0.037 & 4.623 & 0.052 \\
\hline & & Aristidi et al. (1999) & 12.471 & 0.103 & 4.120 & 0.125 \\
\hline & & Docobo \& Ling (1998a) & 15.360 & 0.043 & 5.216 & 0.061 \\
\hline & & Baize (1993c) & 78.081 & 0.128 & 20.149 & 0.165 \\
\hline \multirow[t]{3}{*}{$20151+3742$} & 2416 & Docobo \& Ling (this paper) & 3.634 & 0.026 & 1.812 & 0.027 \\
\hline & & Manté (1999a) & 15.019 & 0.046 & 4.833 & 0.042 \\
\hline & & Couteau (1999a) & 5.167 & 0.024 & 2.409 & 0.026 \\
\hline \multirow[t]{3}{*}{$20216+1930$} & $327 \mathrm{AB}$ & Docobo \& Ling (this paper) & 26.502 & 0.024 & 5.237 & 0.039 \\
\hline & & Docobo \& Ling (2001b) & 18.525 & 0.021 & 4.033 & 0.044 \\
\hline & & Tokovinin (1994) & 32.710 & 0.020 & 4.905 & 0.039 \\
\hline \multirow[t]{2}{*}{$21593+4606$} & 2138 & Docobo \& Ling (this paper) & 9.091 & 0.029 & 4.965 & 0.031 \\
\hline & & Manté (2002) & 7.689 & 0.034 & 4.543 & 0.042 \\
\hline \multirow[t]{2}{*}{$22077+2622$} & 537 & Docobo \& Ling (this paper) & 9.041 & 0.033 & 2.528 & 0.042 \\
\hline & & Baize $(1993 b)$ & 68.598 & 0.031 & 12.542 & 0.042 \\
\hline \multirow[t]{2}{*}{$22307+1758$} & 234 & Docobo \& Ling (this paper) & 6.546 & 0.027 & 2.437 & 0.043 \\
\hline & & Baize (1993a) & 58.008 & 0.059 & 7.766 & 0.060 \\
\hline \multirow[t]{2}{*}{$23199+2844$} & 439 & Docobo \& Ling (this paper) & 5.078 & 0.022 & 1.430 & 0.034 \\
\hline & & Baize (1993b) & 7.126 & 0.044 & 1.935 & 0.075 \\
\hline
\end{tabular}


J. A. Docobo and J. F. Ling: Orbits and masses of 20 double stars discovered by Couteau, Online Material p 6
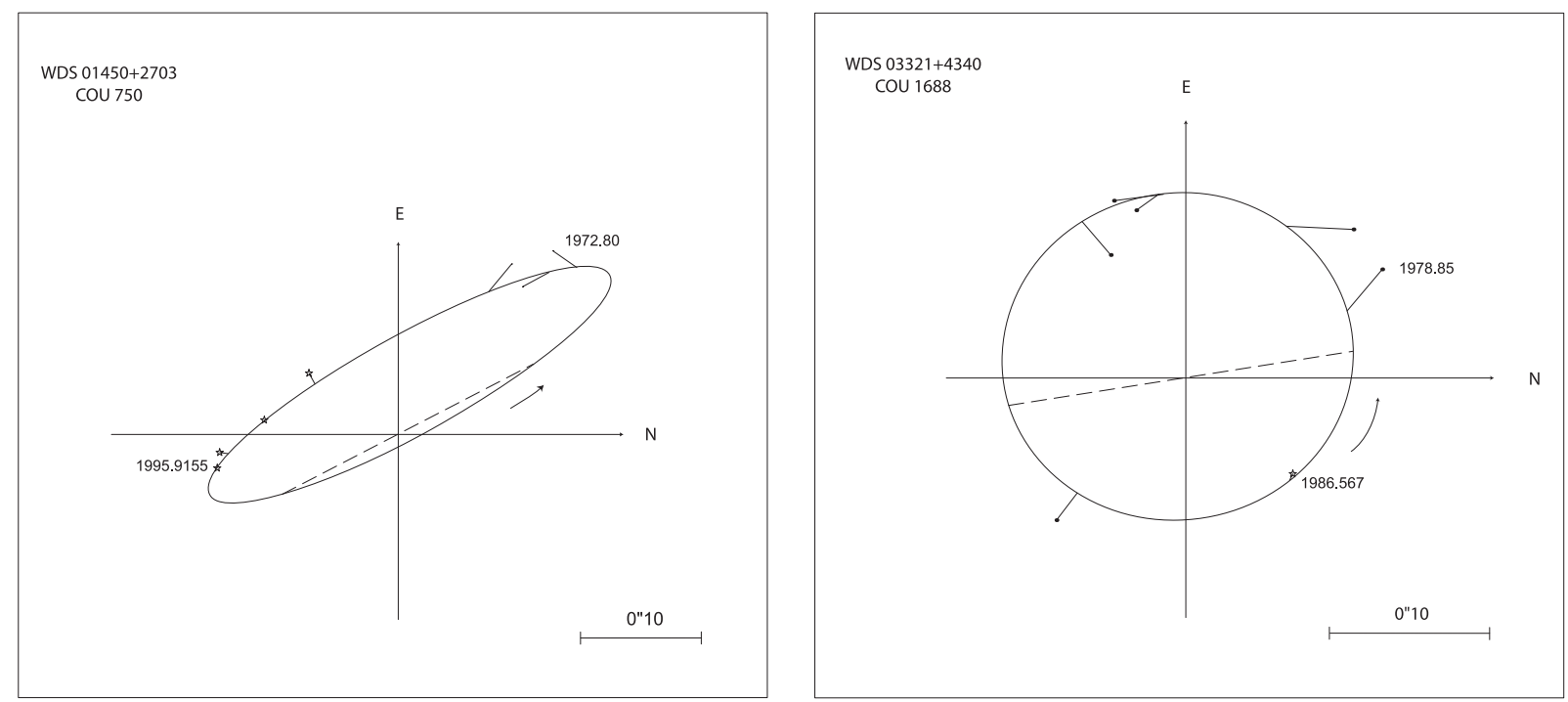

Fig. 1. Apparent orbits for COU 750 and COU 1688.
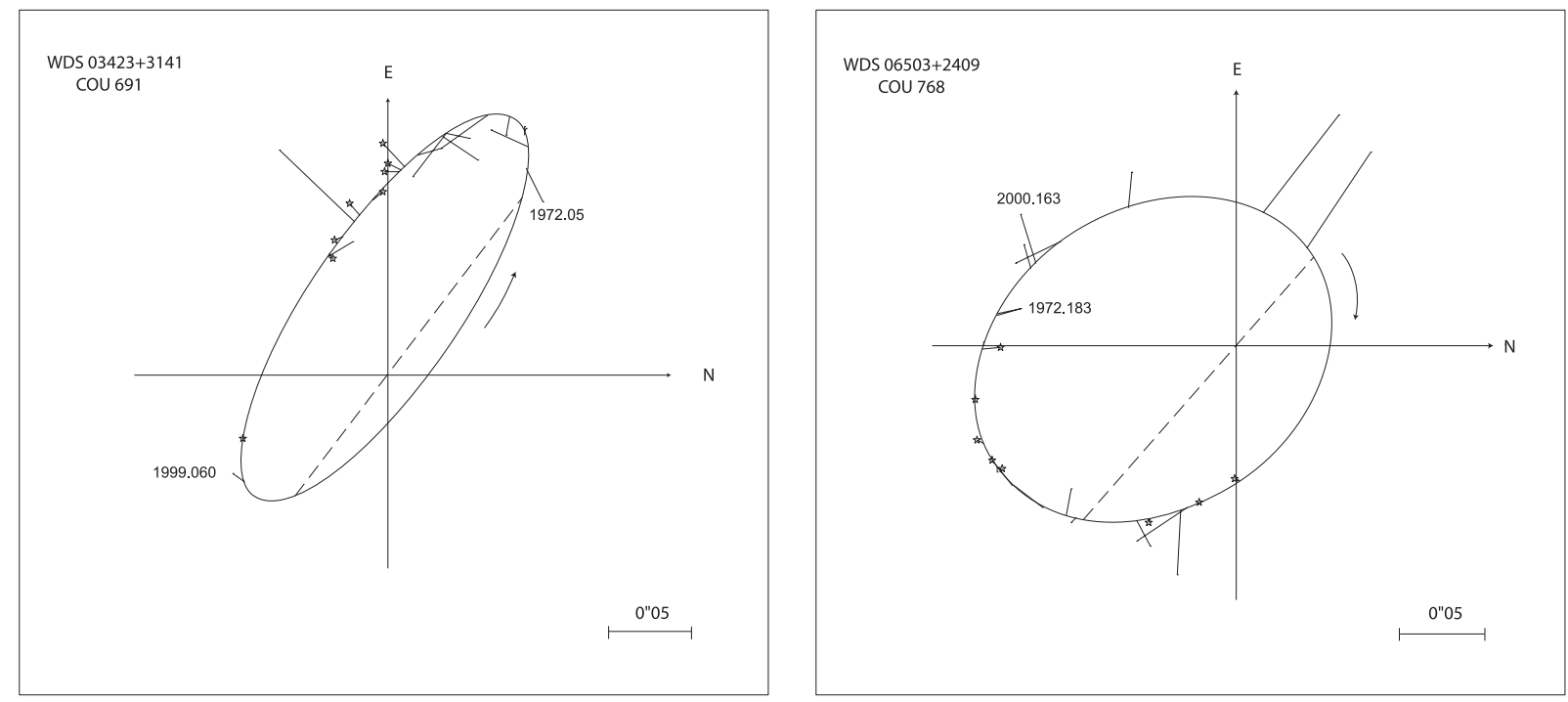

Fig. 2. Apparent orbits for COU 691 and COU 768.
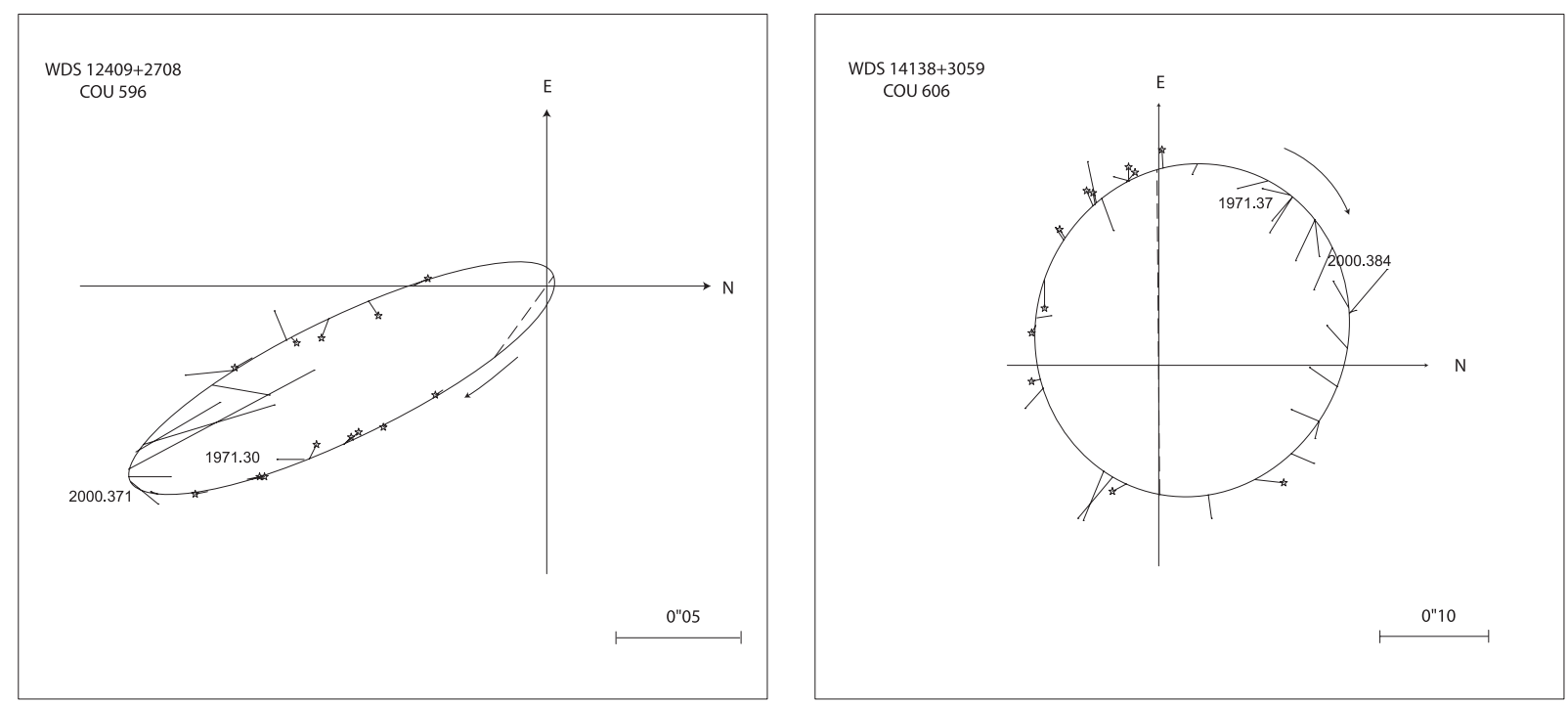

Fig. 3. Apparent orbits for COU 596 and COU 606. 
J. A. Docobo and J. F. Ling: Orbits and masses of 20 double stars discovered by Couteau, Online Material $p 7$
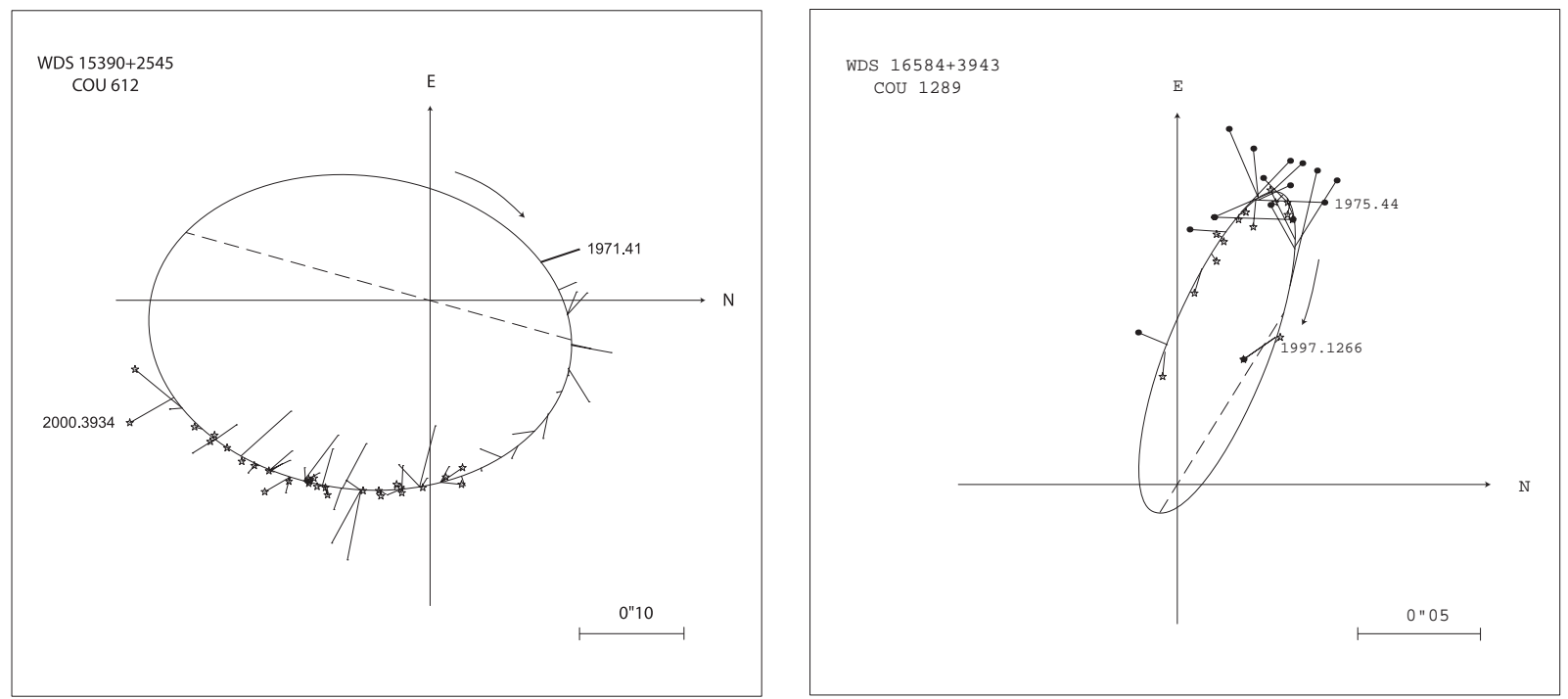

Fig. 4. Apparent orbits for COU 612 and COU 1289.
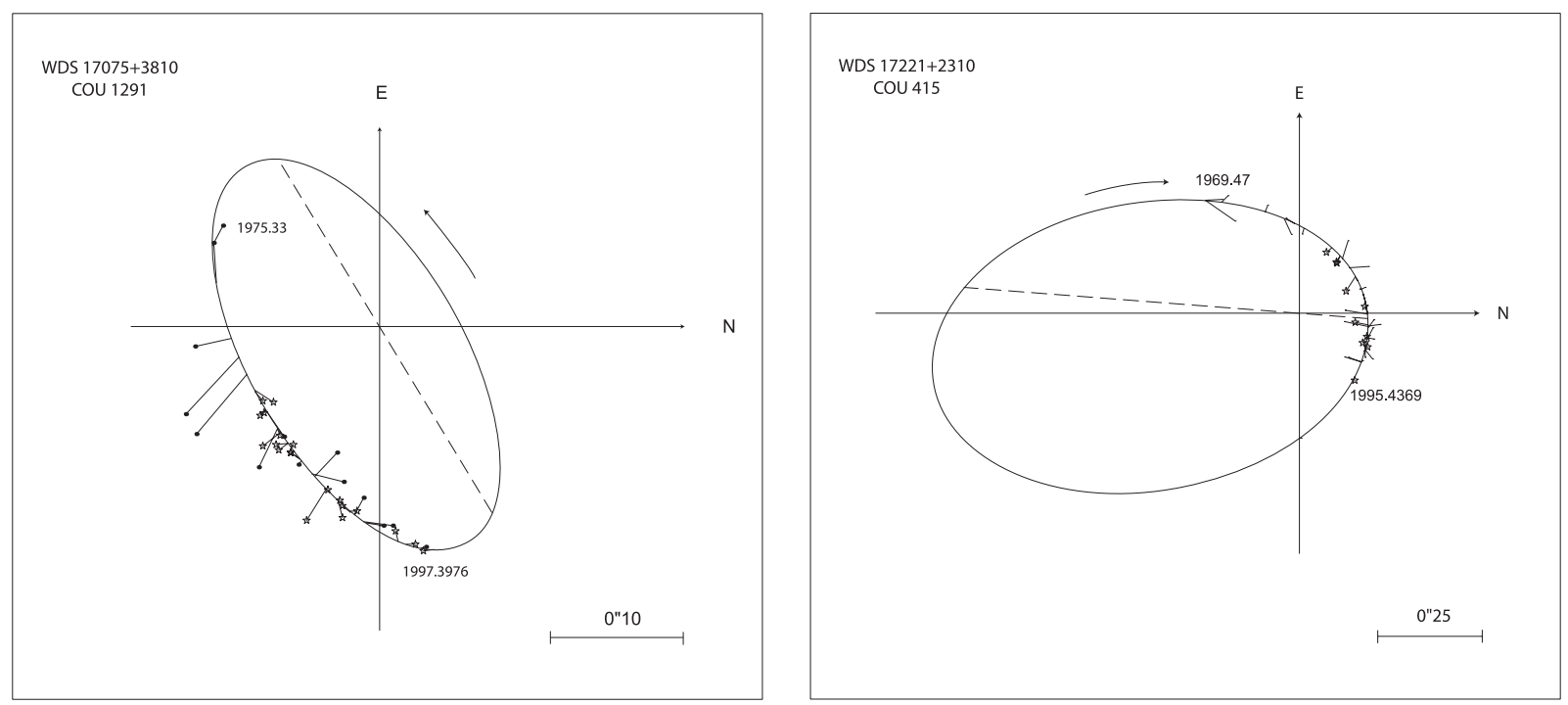

Fig. 5. Apparent orbits for COU 1291 and COU 415.
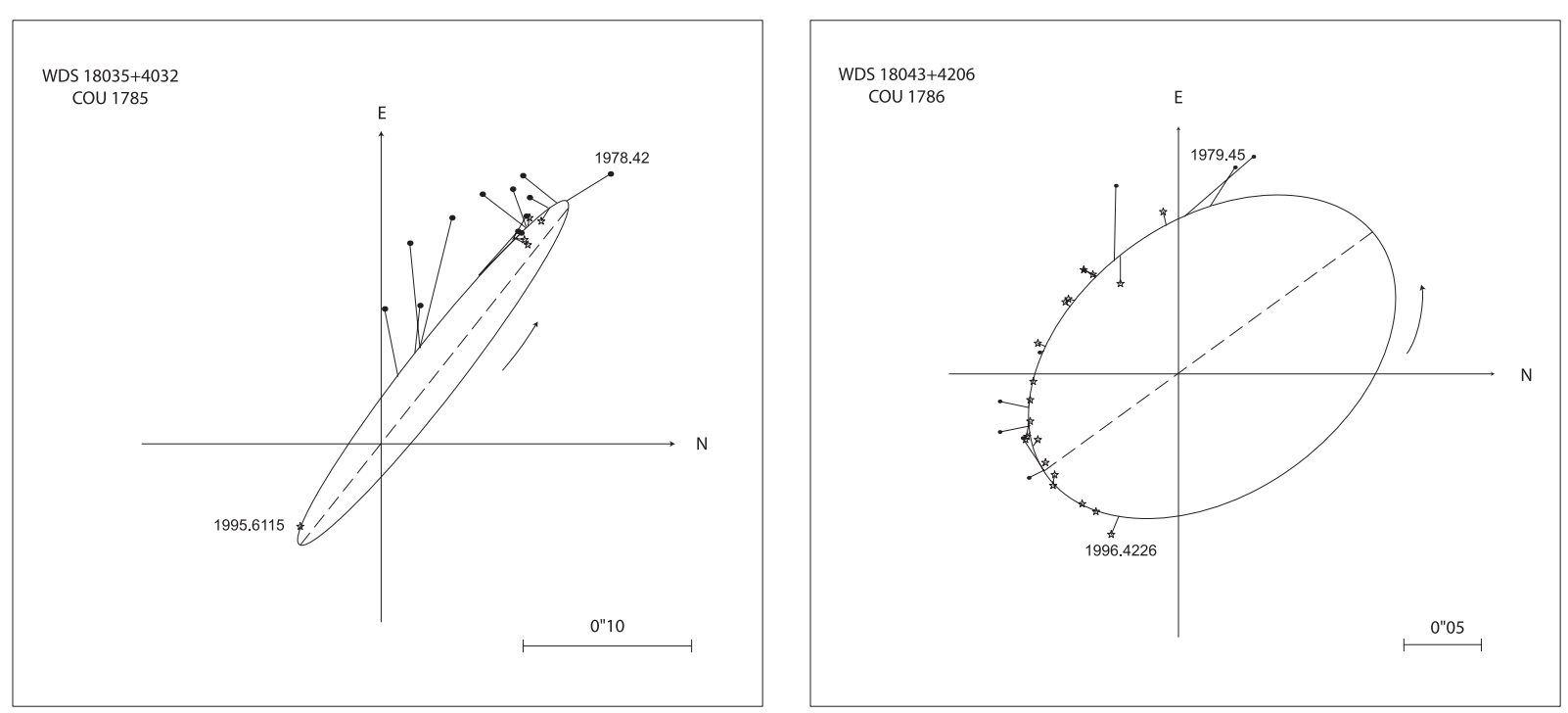

Fig. 6. Apparent orbits for COU 1785 and COU 1786. 
J. A. Docobo and J. F. Ling: Orbits and masses of 20 double stars discovered by Couteau, Online Material p 8
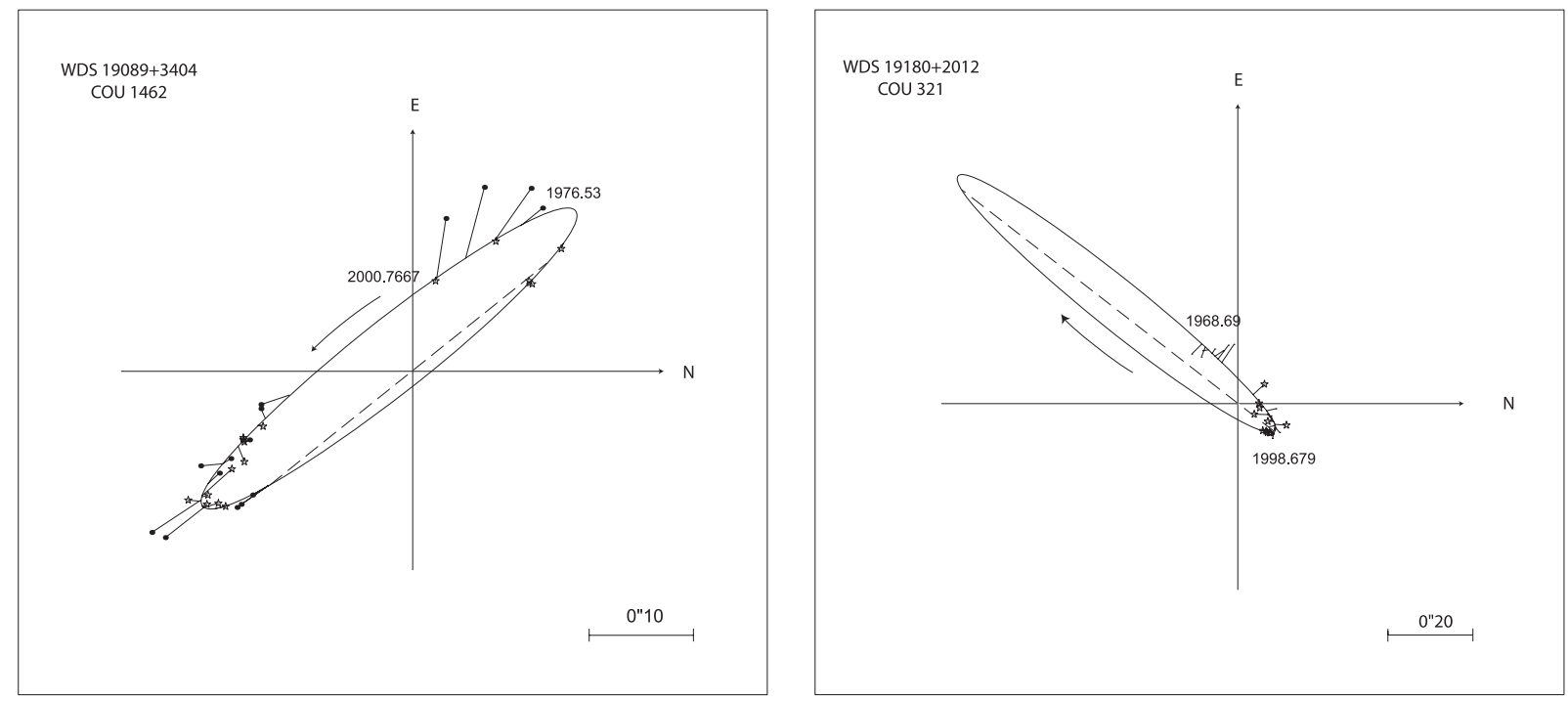

Fig. 7. Apparent orbits for COU 1462 and COU 321
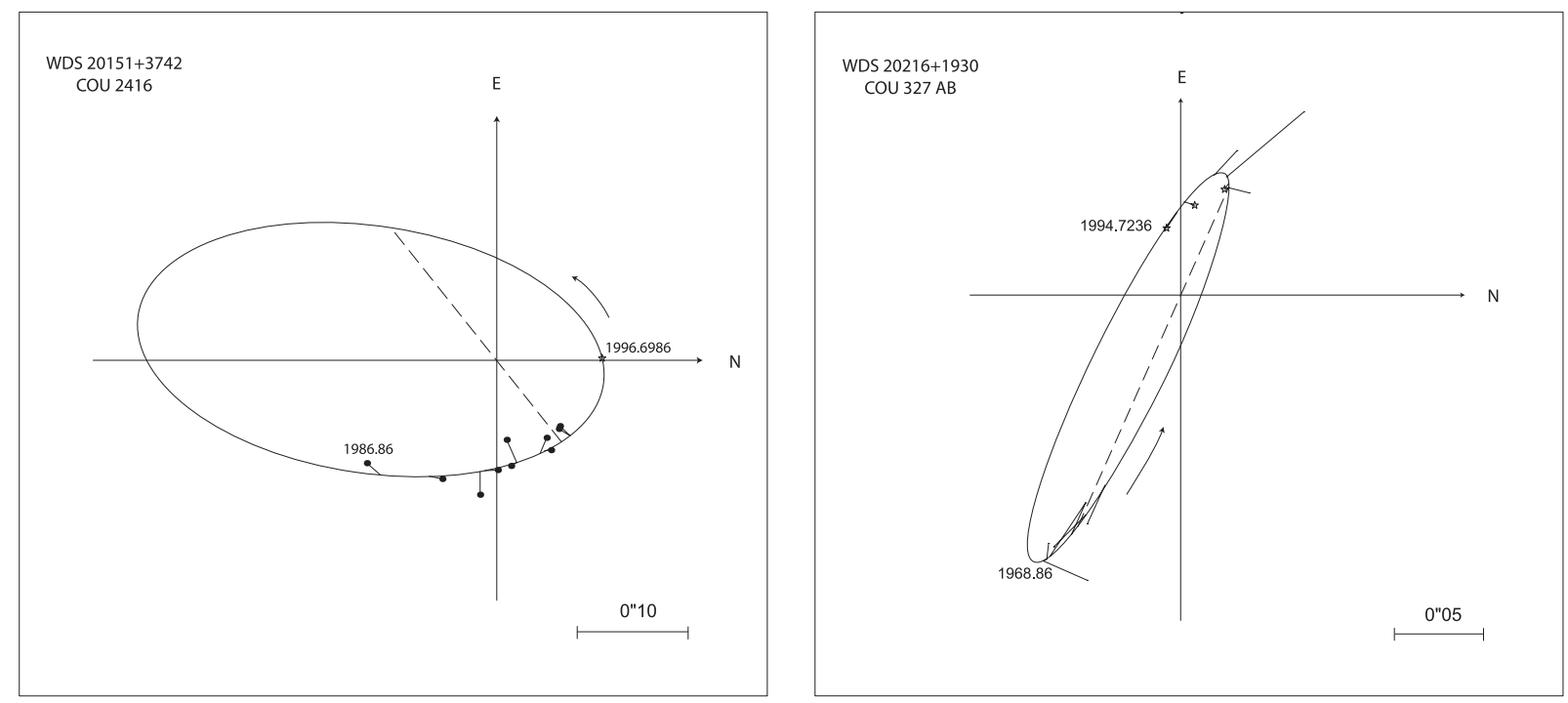

Fig. 8. Apparent orbits for COU 2416 and COU 327 AB.
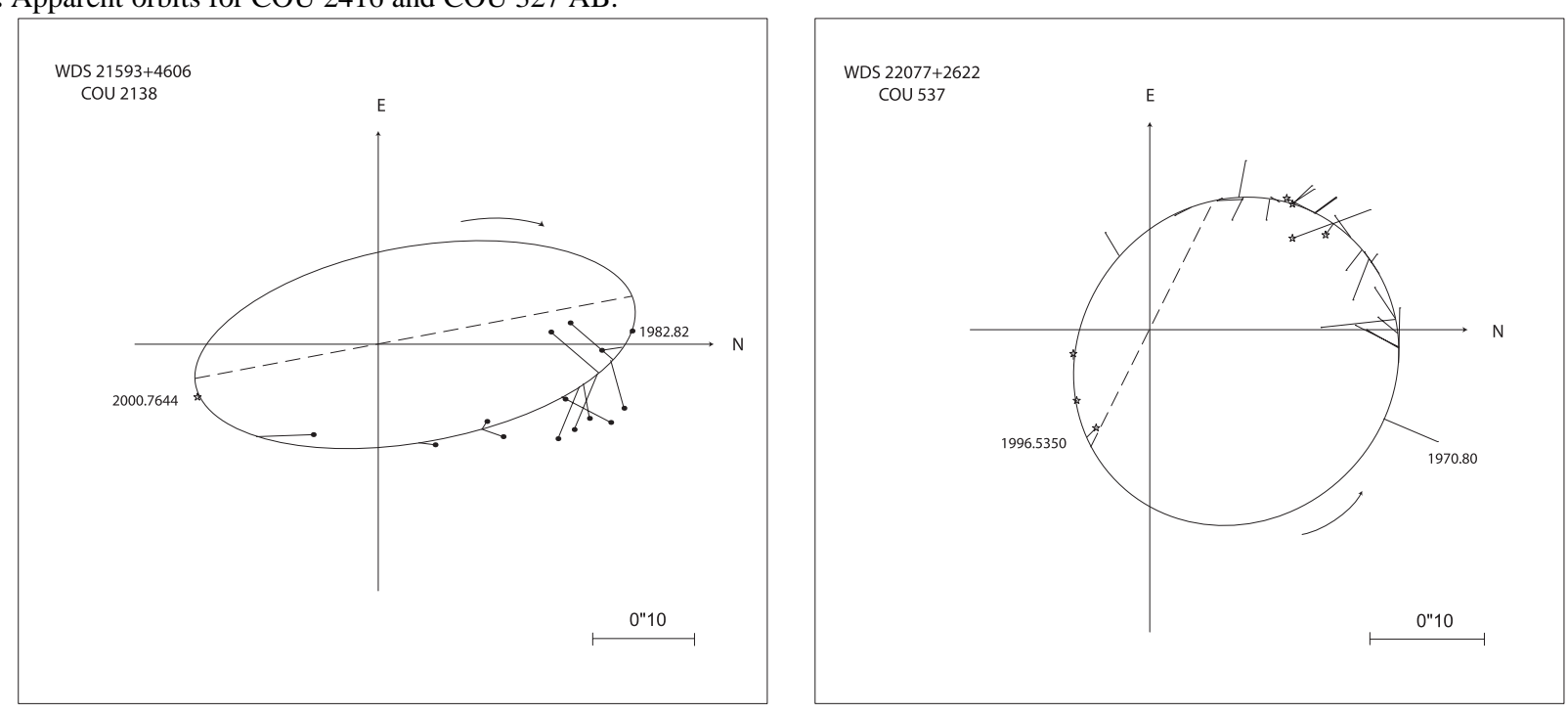

Fig. 9. Apparent orbits for COU 2138 and COU 537. 
J. A. Docobo and J. F. Ling: Orbits and masses of 20 double stars discovered by Couteau, Online Material $p 9$
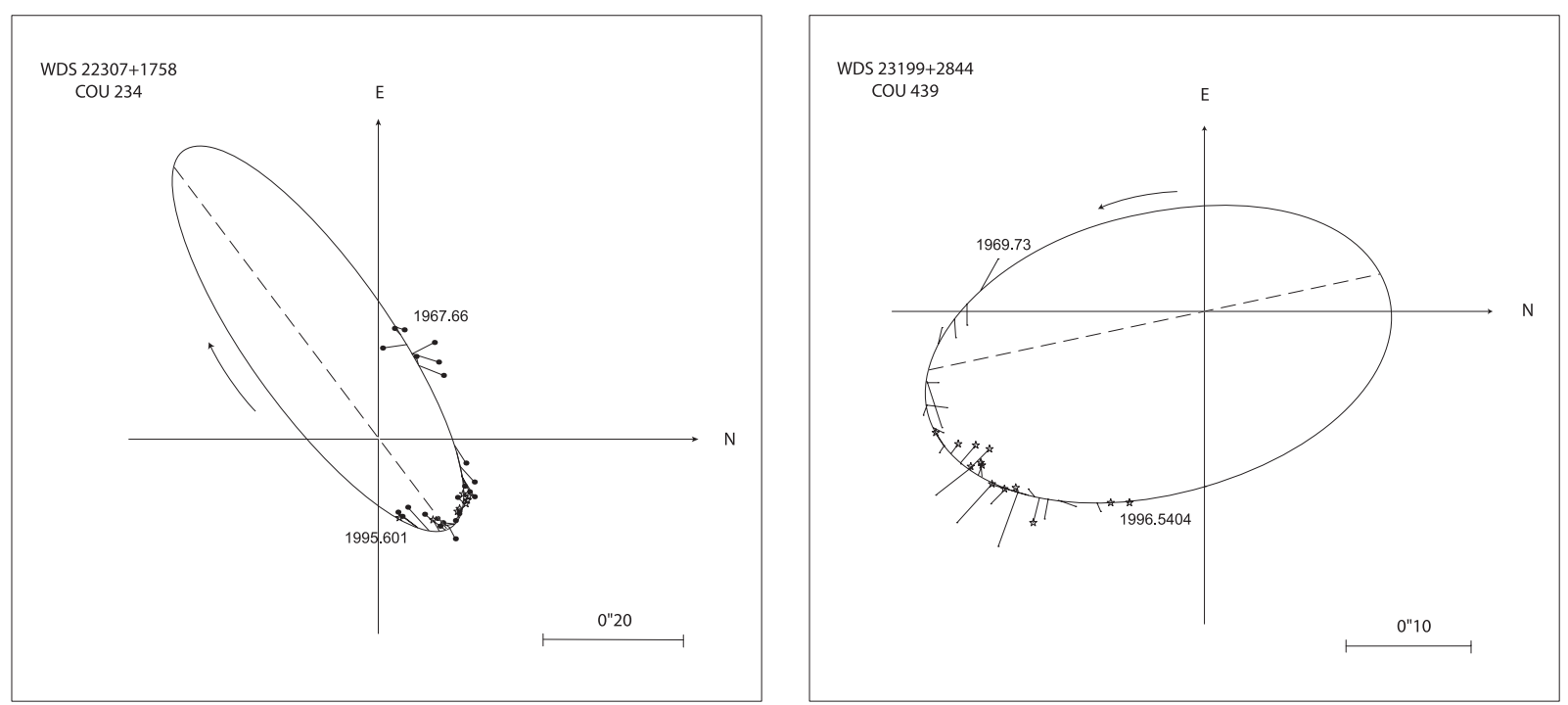

Fig. 10. Apparent orbits for COU 234 and COU 439. 